\title{
A comparative molecular dynamics study of crystalline, paracrystalline and amorphous states of cellulose
}

\author{
Karol Kulasinski • Sinan Keten • \\ Sergey V. Churakov $\cdot$ Dominique Derome • \\ Jan Carmeliet
}

Received: 4 November 2013/Accepted: 20 February 2014/Published online: 1 March 2014

(C) Springer Science+Business Media Dordrecht 2014

\begin{abstract}
The quintessential form of cellulose in wood consists of microfibrils that have high aspect ratio crystalline domains embedded within an amorphous cellulose domain. In this study, we apply unitedatom molecular dynamics simulations to quantify changes in different morphologies of cellulose. We compare the structure of crystalline cellulose with paracrystalline and amorphous phases that are both obtained by high temperature equilibration followed by quenching at room temperature. Our study reveals that the paracrystalline phase may be an intermediate, kinetically arrested phase formed upon amorphisation of crystalline cellulose. The quenched structures yield isotropic amorphous polymer domains consistent with experimental results, thereby validating a new
\end{abstract}

Electronic supplementary material The online version of this article (doi:10.1007/s10570-014-0213-7) contains supplementary material, which is available to authorized users.

K. Kulasinski $(\bowtie) \cdot$ D. Derome · J. Carmeliet

Laboratory for Building Science and Technology, EMPA, Dübendorf, Switzerland

e-mail: kulasinski@arch.ethz.ch

\section{K. Kulasinski}

Department of Civil, Environmental and Geomatic

Engineering, ETH Zürich, Zürich, Switzerland

\section{S. Keten $(\square)$}

Department of Civil and Environmental Engineering,

Northwestern University, 2145 Sheridan Road, Evanston,

IL 60208-3109, USA

e-mail: s-keten@northwestern.edu computational protocol for achieving amorphous cellulose structure. The non-crystalline cellulose compared to crystalline structure is characterized by a dramatic decrease in elastic modulus, thermal expansion coefficient, bond energies, and number of hydrogen bonds. Analysis of the lattice parameters shows that I $\beta$ cellulose undergoes a phase transition into hightemperature phase in the range of $450-550 \mathrm{~K}$. The mechanisms of the phase transition elucidated here present an atomistic view of the temperature dependent dynamic structure and mechanical properties of cellulose. The paracrystalline state of cellulose exhibits intermediate mechanical properties, between crystalline and amorphous phases, that can be assigned to the physical properties of the interphase regions between crystalline and amorphous cellulose in wood microfibrils. Our results suggest an atomistic structural view of amorphous cellulose which is consistent with

\author{
S. Keten \\ Department of Mechanical Engineering, \\ Northwestern University, 2145 Sheridan Road, Evanston, \\ IL 60208-3109, USA \\ S. V. Churakov \\ Laboratory for Waste Management, Paul Scherrer \\ Institute, 5232 Villigen, Switzerland \\ J. Carmeliet \\ Chair of Building Physics, ETH Zürich, \\ Zürich, Switzerland
}


experimental data available up to date and provide a basis for future multi-scale models for wood microfibrils and all-cellulose nanocomposites.

Keywords Cellulose - Amorphous cellulose · Paracrystalline $\cdot$ Phase transition

\section{Introduction}

Cellulose constitutes roughly half of all plant structural material when considered in dry mass, which makes it the most abundant biopolymer on earth (Dinwoodie and Building Research Establishment 2000). Furthermore, cellulose is the main polysaccharide used in numerous industries, with broad applications ranging from drug delivery to packaging (Moon et al. 2011). Its broad use arises partly from its abundance, low cost and extraordinary mechanical properties, mainly high Young's modulus, ranging from 100 to $150 \mathrm{GPa}$ (Eichhorn 2012; Tashiro and Kobayashi 1991) comparable to Kevlar in crystalline form. The main source of cellulose is wood pulp, chemically or mechanically separated wood fibres. In wood, cellulose crystals are aggregated into microfibrils. Wood microfibrils are $3 \mathrm{~nm}$ by $3 \mathrm{~nm}$ in cross section and up to few micrometer in length (Andersson et al. 2003). The outer regions of wood microfibrils are strongly disordered, mostly due to the direct contact with hemicellulose, which is largely amorphous in nature. These regions can show the form of paracrystalline or fully amorphous cellulose. The crystallinity of microfibrils ranges from 40 to $60 \%$ in softwood (Andersson et al. 2003; Agarwal 2006). In general, the amorphous to crystalline ratio is a function of the microfibril cross section (Cheng et al. 2009; Eichhorn and Young 2001; Tanpichai et al. 2012; Eichhorn et al. 2010) and can be related to the surface to volume ratio of a microfibril. Mechanical properties of microfibrils, such as elastic modulus or tensile strength, depend strongly on crystallinity and organization of both cellulose phases (Sinko et al. 2013). Therefore, in order to properly model the behavior of microfibrils, nanostructural features and mechanical properties for both the crystalline and amorphous cellulose phases need to be established.

The structure of native cellulose crystals, either I $\alpha$ or $\mathrm{I} \beta$, is well studied and has been characterized through NMR, X-ray scattering experiments, electron diffraction and neutron diffraction methods (Hardy and Sarko 1996; Chen et al. 2012; Nishiyama 2009; Nishiyama et al. 2002a, b, 2003; Sugiyama et al. 1991a, b). Within a crystal, the cellulose chains are organized in sheets, kept together by Van der Waals and weak C...H and O...H hydrogen-bonding interactions. Within a sheet, the cellulose chains are stabilized by numerous hydrogen bonds between the glucose hydroxyl groups.

I $\beta$ crystalline phase is the most common form of cellulose, present in large quantities in plant cells, thus this form has been widely studied both by experiments and molecular dynamics (MD) modeling using the GROMOS force field (Charlier and Mazeau 2012; Chen et al. 2012; Bergenstrahle et al. 2007, 2008a; Matthews et al. 2012; Wohlert et al. 2012; Bergenstrahle et al. 2010; Hadden et al.; Zhang et al. 2011; Chen et al. 2013). MD simulations provide molecular level insight into the cellulose structure, its organization, surface and interfacial properties that complement experimental studies.

In contrast to crystalline cellulose, there are numerous aspects pertaining to the structure and properties of amorphous cellulose that are relatively less understood. Experiments suggest that the amorphous state is characterized by an absence of longrange ordering and greater orientation disorder of cellulose chains (Fink et al. 1987; Muller et al. 2000). Transitions from crystalline to amorphous phase have been obtained experimentally, for instance, by boiling native cellulose in water bath at $320{ }^{\circ} \mathrm{C}$ under $25 \mathrm{MPa}$ (Deguchi et al. 2006, 2008). However, the changes in the major structural characteristics of cellulose such as the occupancy of hydrogen bonds, bond energies, and structural morphology during the amorphisation have not been understood so far.

Whereas the structure and mechanical properties of crystalline cellulose are relatively well understood, the properties of paracrystalline interphase regions within microfibrils and the fully amorphous domains remain poorly characterized both experimentally and theoretically. Moreover, the nature and the physical properties of the paracrystalline, not fully amorphized phase, which are essential for understanding the mechanical properties special arrangement of wood microfibrils, have not been explained at molecular level yet.

To address these issues, here we investigate the differences in structural and mechanical properties of crystalline, paracrystalline and amorphous cellulose states. 
We note that cellulose I $\beta$ (Bergenstrahle et al. 2007; Matthews et al. 2006; Mazeau and Heux 2003; Bergenstrahle et al. 2008b) and amorphous cellulose have been modeled and studied by MD simulations in earlier studies (Mazeau and Heux 2003; Liao et al. 2012; Chen et al. 2004a, b, 2007). However, here we present a computational approach to obtaining wellequilibrated amorphous structures and present a new analysis comparing amorphous cellulose properties with available experimental results and the characteristics of crystalline cellulose (Derome et al. 2012). Moreover, we demonstrate that cellulose I $\beta$ undergoes a phase transition at $500 \mathrm{~K}$, confirming experimental observations (Wada et al. 2001). The purpose of this study is to investigate and understand the physical properties of the key constituents of a wood microfibril, i.e. crystalline, paracrystalline and amorphous celluloses, through a consistent and comparative simulation approach. Moreover, this research will help determine whether the non-amorphous regions of cellulose microfibrils are paracrystalline or fully amorphous.

\section{Materials and methods}

All the simulations are carried out using GROMACS 4.6 software (Hess et al. 2008) and GROMOS 53a6 united atom force field (Oostenbrink et al. 2004; Lins and Hünenberger 2005), where an aliphatic carbon together with its hydrogen(s) is represented by one bead. The cut-off radii of Coulomb and Van der Waals forces are set to $1.0 \mathrm{~nm}$, long-range Coulomb interactions are accounted for by Particle-Mesh Ewald summation, the energy and pressure are corrected for dispersion forces, and the $\mathrm{OH}$ bonds are constrained. The initial coordinates of cellulose I $\beta$ crystal structure have been generated by a script (Gomes and Skaf 2012) using the most recent crystallographic structure reported by Nishiyama et al. (2002b). The equation of motions are integrated with a 1 fs time step and the coupling methods for temperature and pressure are velocity-rescaling (Bussi et al. 2007) and Berendsen barostat (Berendsen et al. 1984), respectively. Before equilibration, a steepest descent followed by conjugated gradients energy minimization method is performed. The values of compressibility for crystalline cellulose are as follows: $(2.5 \mathrm{e}-5,2.2 \mathrm{e}-5,7.9 \mathrm{e}-7$, $1.2 \mathrm{e}-5,4.2 \mathrm{e}-5,4.4 \mathrm{e}-5)$ for crystalline, $(2.0 \mathrm{e}-5$, $2.0 \mathrm{e}-5, \quad 7.9 \mathrm{e}-7, \quad 1.0 \mathrm{e}-5, \quad 1.0 \mathrm{e}-5,1.0 \mathrm{e}-5)$ for paracrystalline, and $(2.0 \mathrm{e}-5,2.0 \mathrm{e}-5,2.0 \mathrm{e}-5,1.0 \mathrm{e}-$ $5,1.0 \mathrm{e}-5,1.0 \mathrm{e}-5)$ for amorphous cellulose.

Our model of crystalline cellulose consists of 32 cellulose chains, ten cellobiose units long, with periodic boundary conditions and covalently bonded across the periodic boundary, in order to create an infinite crystal without defects. We note that the constructed crystalline cellulose is a model without defects. However, both introducing some defects and dealing with finite-size crystal will increase the pace of amorphisation, as the process of amorphisation always starts from defects, e.g. chain ends. We expect that introducing defects will increase potential energy terms and decrease the elastic modulus of the crystalline cellulose.

The simulation box is first equilibrated for $10 \mathrm{~ns}$ in isobaric-isothermal (NPT) ensemble at 1 bar and $300 \mathrm{~K}$. The simulated crystalline structure is presented in Fig. 1a. Figure 2a defines the lattice parameters of crystalline cellulose: $a$, twice the intersheet distance; $b$, the chain-to-chain distance within a sheet; $c$, corresponding to the length of cellobiose unit; $\alpha$, the tilt of the cellulose sheets in the plane parallel to the chain direction; $\beta$, the angle between the cellulose chains within a sheet; and $\gamma$, the tilt of the cellulose sheets in the cross section perpendicular to the chain direction. The key parameters of the simulated structure are presented in Table 1.

In order to investigate the influence of temperature on the crystal structure and long-range ordering, in the crystalline I $\beta$ cellulose, the system is gradually heated from 250 to $650 \mathrm{~K}$ in $50 \mathrm{~K}$ steps, equilibrating the system in NPT ensemble for $1 \mathrm{~ns}$ at each temperature, resulting in $9 \mathrm{~ns}$ of the total simulation time. The simulation time was enough to equilibrate pressure and system volume. An anisotropic pressure barostat is applied, such that all 6 components of the stress matrix are varied independently.

The paracrystalline state is obtained from the crystalline structure by heating the crystalline cellulose structure at $700 \mathrm{~K}$ until it loses its ordering, typically after $1 \mathrm{~ns}$. This step is followed by a quenching simulation at $300 \mathrm{~K}$ for $1 \mathrm{~ns}$, with an intermediate simulation at $500 \mathrm{~K}$ for $100 \mathrm{ps} .700 \mathrm{~K}$ is the minimum temperature at which crystalline cellulose loses its ordered structure. At higher temperatures, a similar effect is expected but the process of disordering will be accelerated. $300 \mathrm{~K}$ is chosen to study the properties of different cellulose forms because it is most relevant for material applications 

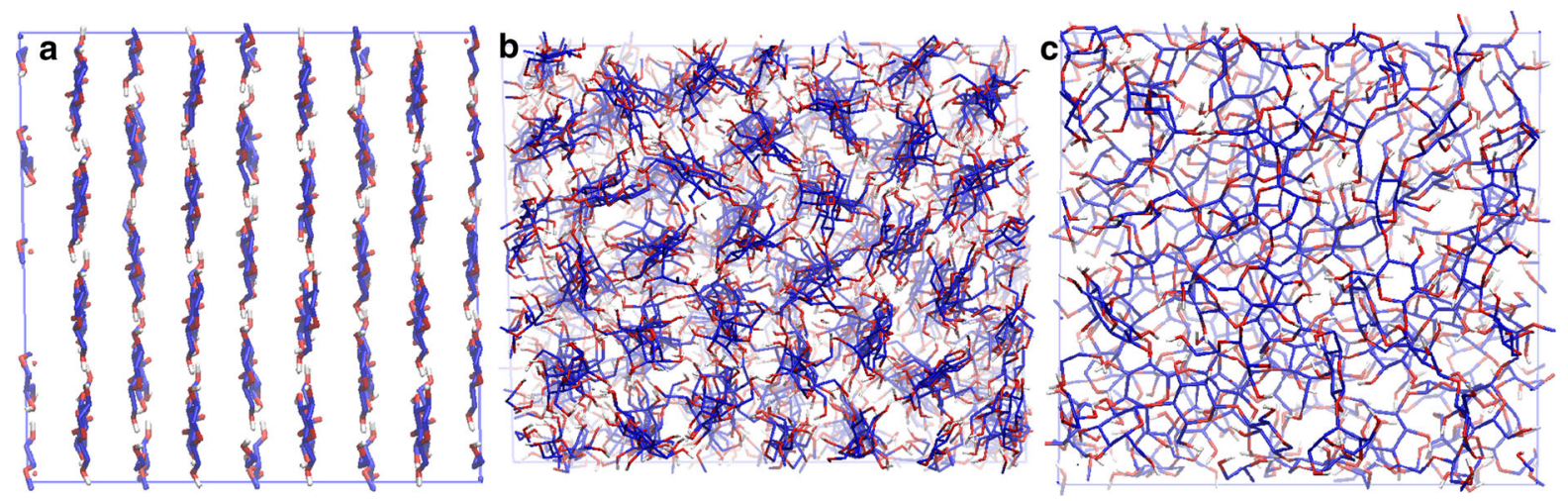

Fig. 1 Atomistic structure and simulation box of crystalline I $\beta$ (a) paracrystalline (b) and amorphous (c) cellulose

and also the standard temperature in experiments. To obtain the paracrystalline state, we rely on the caging effects of other chains, which restrict the relaxation of the cellulose chains and prevent complete disordering. The final paracrystalline structure obtained through this procedure is shown in Fig. 1b.

In order to obtain a representative amorphous cellulose model, we build 5 initial structures that consist of 2-4 cellulose chains with the degree of polymerization (DP) ranging from 10 to 40, and large interchain spacing. The 5 built structures have the following parameters: four chains of $\mathrm{DP}=20$ with parallel chains arrangement, four chains of DP $=20$ and random arrangement, four chains of DP $=10$ and parallel arrangement, three chains of DP $=30$ and random arrangement, two chains of DP $=40$ and random arrangement. Large spacing between the chains and high temperature enables free twisting and folding of the cellulose chains, which leads to the loss of long-range ordering and formation of an amorphous isotropic phase. The obtained isotropic amorphous phase is then quenched and equilibrated at $300 \mathrm{~K}$ and 1 bar until the potential energy and the cell volume are fluctuating around a constant value. The key metric that we use for assessing the amorphous phase is density, which is typically 10-20\% smaller than the density of the crystalline phase (Mazeau and Heux 2003). The amorphous cellulose average density obtained in our simulations is $1.285 \mathrm{~g} \mathrm{~cm}^{-3}$ (Table 1), which is $14 \%$ less than crystalline phase density and agrees with the general trend mentioned above. The resulting amorphous structure is presented in Fig. 1c.

In order to calculate the elastic modulus, the system is simulated in NPT ensemble with anisotropic barostat. A constant stress in a given direction is imposed and the strain is measured once the system has reached the equilibrium state. For each direction, at least three stress-strain points are calculated. The anisotropic expansion coefficients are calculated by changing the temperature in the range $200-400 \mathrm{~K}$ in $50 \mathrm{~K}$ increments and measuring the changes in box lengths, while keeping the pressure at 0 bar.

The criterion of hydrogen bond existence combines a cut-off radius of $0.35 \mathrm{~nm}$ between donor and acceptor (corresponding to the first minimum of the radial distribution function of SPC water (Apol et al. 2010)) and a hydrogen-donor-acceptor angle with a cutoff of $30^{\circ}$. The hydrogen bond pattern considered here is pattern A, by far the most common, as specified in (Nishiyama et al. 2002b). The tool used to determine hydrogen bonding existence is 'g_hbond' and the puckering parameters are determined with 'g_puckering', both provided with GROMACS package.

\section{Results}

Temperature phase transition of crystalline cellulose

We first investigate the effect of temperature on the crystal structure and long-range ordering of the crystalline cellulose as shown in Fig. 2. First, looking at the characteristic angles, Fig. 2b shows that $\alpha$ and $\beta$ remain constant over the wide range of temperatures. In contrast, $\gamma$, that defines shear of the unit cell, has undergone a drastic change from $91^{\circ}$ down to $70^{\circ}$ in the temperature range $450-550 \mathrm{~K}$, indicating that 
Fig. 2 a Crystal structure of the crystalline I $\beta$ cellulose. Temperature dependence of $\mathbf{b}$ the lattice angles and $\mathbf{c}$ lattice dimensions, $\mathbf{d}$ density and unit cell volume, e hydrogen bonds per glucose unit and hydrogen bonds density. A Transition from I $\beta$ to high-temperature phase of the crystalline cellulose occurs in the temperature range $450-550 \mathrm{~K}$

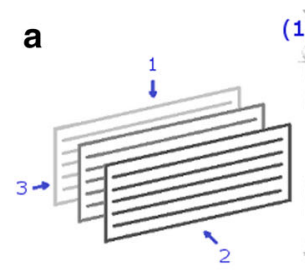

(1)
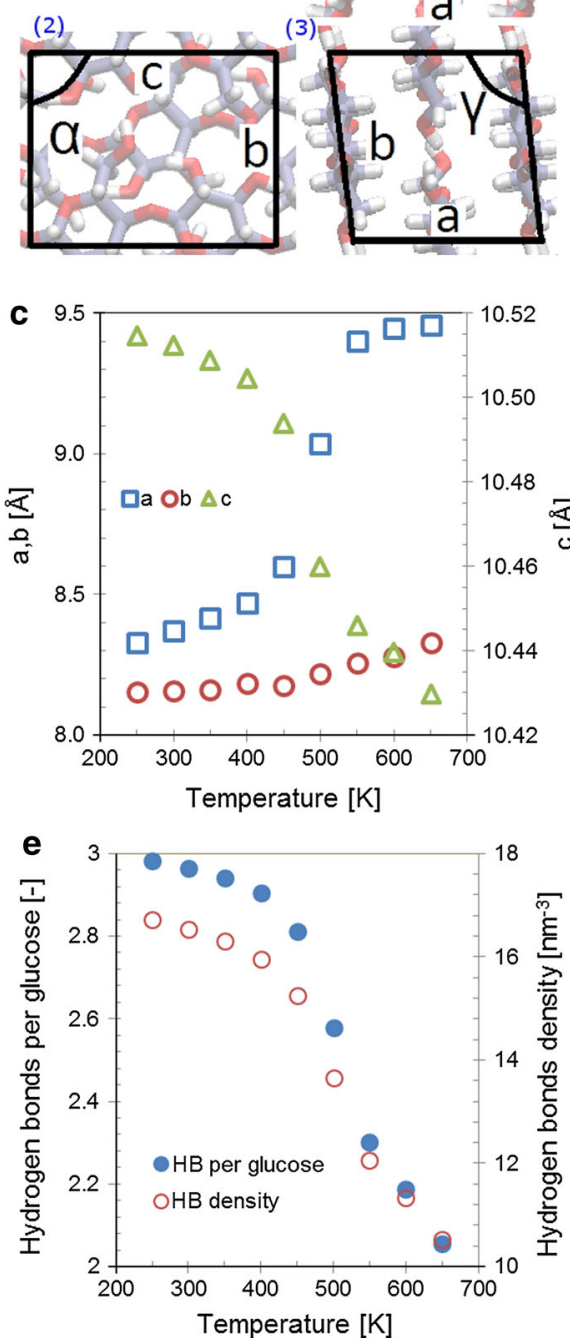

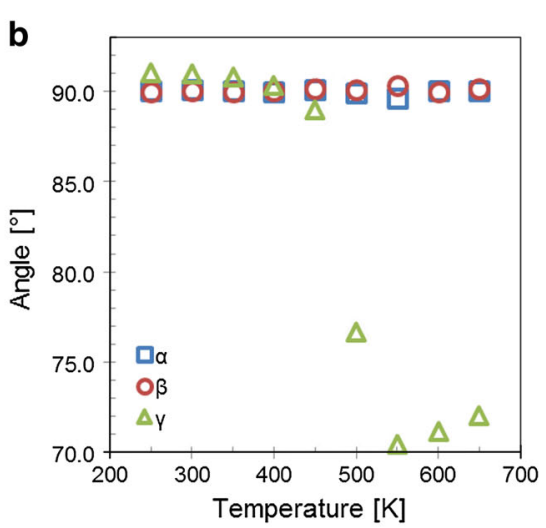

d

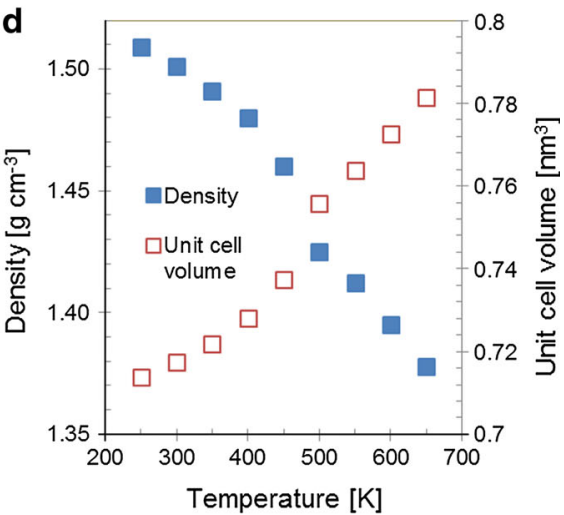

Table 1 Experimentally measured (Nishiyama et al. 2002b) and simulated density and lattice parameters of I $\beta$ crystalline cellulose

\begin{tabular}{llllll}
\hline Cell parameters & $\rho\left(\mathrm{g} \mathrm{cm}^{-3}\right)$ & $\mathrm{a}(\AA)$ & $\mathrm{b}(\AA)$ & $\mathrm{c}(\AA)$ & $\gamma\left({ }^{\circ}\right)$ \\
\hline Experimental & 1.656 & 7.784 & 8.201 & 10.38 & 96.5 \\
Simulations & 1.501 & $8.37 \pm 0.13$ & $8.16 \pm 0.14$ & $10.51 \pm 0.13$ & $91.0 \pm 0.1$ \\
\hline
\end{tabular}

structural changes are due to the reordering of the hydrogen bonding patterns between the cellulose chains. Looking at the characteristic lattice dimensions (Fig. 2c), in the same temperature interval, the lattice parameter $a$ is found to increase by $9 \%$ and the lattice parameter $b$ to increase by $1 \%$. 
This sharp change in the lattice parameters indicates a phase transition of the I $\beta$ allomorph into hightemperature phase. Such a transition has been previously observed experimentally (Wada et al. 2001), as well as by other MD simulations (Chen et al. 2012; Bergenstrahle et al. 2007).

The cellulose crystals are strongly anisotropic. Whereas the parameter $c$ is shrinking with the temperature, $a$ and $b$ are increasing (Fig. 2c). This can be explained by the fact that $a$ corresponds to the stacking distance between sheets, which interact primarily through weak van der Waals interactions. As such, an increase in temperature results in a greater expansion along this direction. On the other hand, $b$ is increasing, as more hydrogen bonds are broken due to the increasing temperature. The cellulose chains in crystalline phase are stretched in the longitudinal direction, i.e. the harmonic potential of bonds and angles is far from its minimum state, which, in turn, is stabilized by numerous hydrogen bonds between the chains. However, an increase in temperature causes breaking of some hydrogen bonds, mostly intrachain HO3...O5 and HO2..O6, in the phase transition. The shrinkage of chains ( $c$ parameter) can be related to the fact that nonnative celluloses have smaller value of $c$ than native cellulose, while having smaller number of hydrogen bonds (Wada et al. 2004; Langan et al. 1999).

As shown in Fig. 2d, the density of the cellulose decreases from $1.5 \mathrm{~g} \mathrm{~cm}^{-3}$ at $300 \mathrm{~K}$ to $1.38 \mathrm{~g} \mathrm{~cm}^{-3}$ at $650 \mathrm{~K}$, which is well correlated with the increase in unit cell volume (Fig. 2d) and the drop in average number of hydrogen bonds per glucose unit, from 2.97 down to 2.06 (Fig. 2e). This drop is due to the fact that the cellulose chains coil at high temperature due to chain entropy, and lose their well-ordered structure. Similarly, the hydrogen bonds density, which is the total number of hydrogen bonds divided by the system volume, decreases from 18 to nearly $10 \mathrm{~nm}^{-3}$ (Fig. 2e).

The temperature affects also the relative position of hydroxymethyl group (carbon C6), given by the dihedral angle between glucose ring and hydroxymethyl group, $\omega$ (Fig. 3), which is another key characteristic of the conformation adopted by cellulose chains is various phases. The position of the hydroxymethyl group can be trans-gauche (TG), gauche-gauche (GG) or gauche-trans (GT). The torsion angle $\omega$ is affected by temperature as shown in Fig. 3. We find that the TG conformation is the only conformation observed at low temperatures. At $500 \mathrm{~K}$, GG and GT conformations are preferred since they are more favorable energetically according to an MD study (Chen et al. 2012). At $500 \mathrm{~K}$, all the three conformations are observed. Yet at $600 \mathrm{~K}$, at the end of the phase process, only GG and GT are present. Despite the fact that TG is the conformation of the highest dihedral energy (Chen et al. 2012), it facilitates formation of interchain hydrogen bonds, that decrease significantly their occupancy at higher temperatures (Fig. 2d). As the temperature increases, the hydroxymethyl groups start to change from the TG conformation (central peak) to GG and GT conformations (side peaks). The largest structural transition appears in the 450-550 K temperature range, which corroborates well with the trend observed in density and hydrogen bond temperature profiles in Fig. 2.

Dihedral angles and puckering parameters

To compare the crystalline, paracrystalline and amorphous states of cellulose, we first present the distributions of dihedral angles in each cellulose phase in Fig. 4. The chosen dihedral angles are $\omega\left(\mathrm{O}_{5}-\mathrm{C}_{5}-\mathrm{C}_{6}-\right.$ $\mathrm{O}_{6}$ ), corresponding to the position of the hydroxymethyl group, and $\phi\left(\mathrm{O}_{5}-\mathrm{C}_{1}-\mathrm{O}_{4}-\mathrm{C}_{4}{ }^{\prime}\right)$ and $\psi\left(\mathrm{C}_{1}-\mathrm{O}_{4}-\right.$ $\mathrm{C}_{4}{ }^{\prime}-\mathrm{C}_{5}{ }^{\prime}$ ), being the characteristic torsion angles of the glycosidic linkage between the two consecutive glucose rings. The $\omega$ distribution of amorphous cellulose resembles that of crystalline at $600 \mathrm{~K}$, shown in Fig. 3, although the trans-gauche conformation occurs in 7 , versus $3 \%$ for crystalline at $600 \mathrm{~K}$ (almost disappeared). The thermodynamic state of $\omega$ dihedrals in amorphous cellulose can thus be found as a more preferred, lower energy configuration. Our wellrelaxed structure shows clearly the three distinct peaks in the dihedral distribution. On the other hand, the much broader distribution of $\phi$ confirms the nonordered character of amorphous cellulose. The $\omega$ distribution for paracrystalline is identical to that of amorphous cellulose, proving that the relaxation of the cellulose structure at high temperature is enough to reach the lowest energy conformation of the hydroxymethyl group.

Likewise, the distribution of $\Phi$ angles in paracrystalline and amorphous phases are overlapping and show an additional peak at $60^{\circ}$, that is a footprint of an additional, minor mode of glucopyranose ring-to-ring tilt in cellulose chain, and that is shifted by $180^{\circ}$ from 
Fig. 3 Probability density distribution of $\omega$ torsional angle which determines the relative position of the hydroxymethyl group as function of temperature
Fig. 4 Probability density distributions of dihedral $\omega$ (a), $\Phi(\mathbf{b})$ and $\Psi(\mathbf{c})$ angles for crystalline, paracrystalline and amorphous states of cellulose, at $300 \mathrm{~K}$
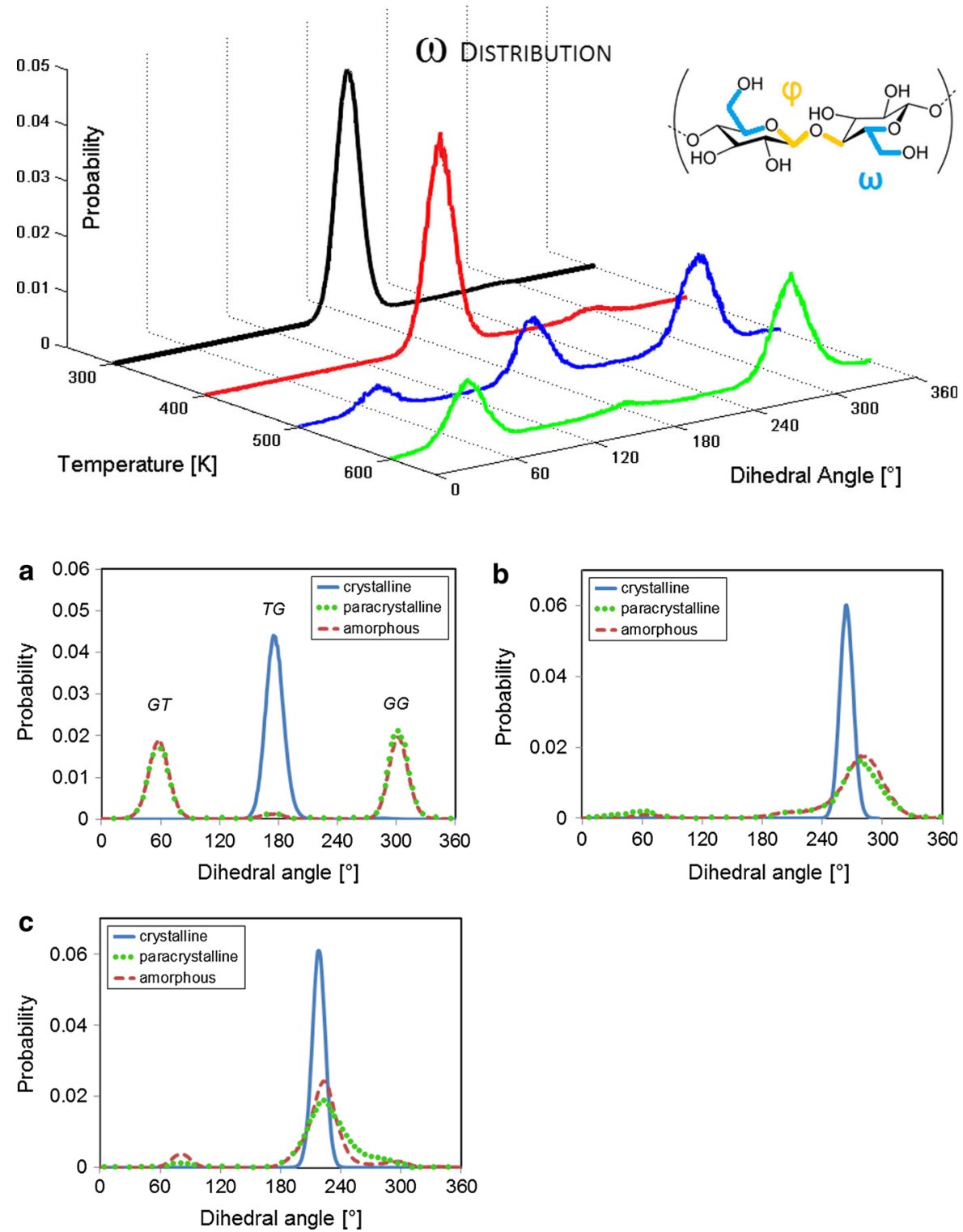

the major peak. The $\Psi$ distribution (Fig. 4c) is similar to the $\Phi$ distributions, with one major peak for crystalline cellulose, broadened peaks for non-crystalline cellulose, as well as a minor, although well distinguished, peak at $90^{\circ}$. This observation has not yet been confirmed experimentally, but provides potentially a new metric that could help to distinguish the noncrystalline phase from other phases of cellulose. In contrast, $\tau$, the glycosidic linkage angle $(\mathrm{C} 1-$ O4-C4), does not vary with phases, as $\tau=117.3^{\circ}$ for crystalline and paracrystalline and $\tau=117.4^{\circ}$ for amorphous cellulose.
Cellulose phase can be characterized also by the puckering parameters, $\phi, \theta$ and $\mathrm{Q}$, of a glucopyranose ring (Cremer and Pople 1975). The puckering parameters define unambiguously the shape of a six-membered ring. The relevance of the distortions of pyranoid rings lies in their influence on the viscoelastic properties of polysaccharides (Heymann and Grubmüller 1999; Rief et al. 1997). The distribution maps of the puckering angles, $\phi$ and $\theta$, for the discussed cellulose phases, are presented in Fig. 5. We do not present here the distribution of the Q parameter, as it does not show any distinct difference between the 

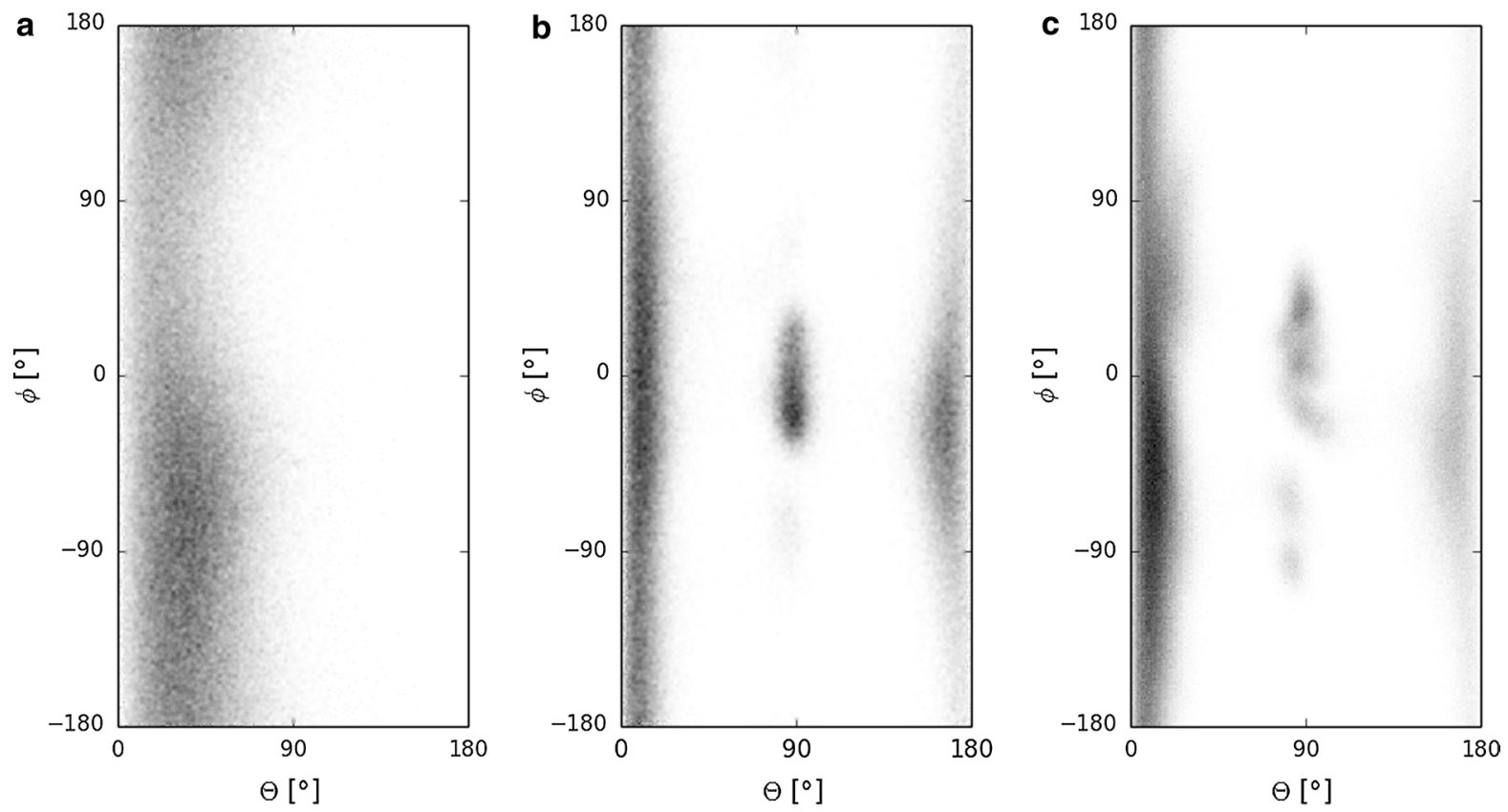

Fig. 5 Puckering parameters, $\phi$ and $\theta$, distribution map for a crystalline, b paracrystalline and $\mathbf{c}$ amorphous cellulose

studied cellulose phases, with an average value of $\mathrm{Q}=0.58 \mathrm{~nm}$. For $\theta=0^{\circ}\left({ }^{4} \mathrm{C}_{1}\right.$ 'chair' $)$, the occurrence of ring puckering is almost equally possible for any value of $\phi$. In contrast to crystalline cellulose, the non-crystalline phases exhibit two additional 'modes', namely for $\theta$ equal $90^{\circ}$ ('boat') and $180^{\circ}$ (1C4 'chair'). The paracrystalline phase has a particularly well pronounced mode at $\phi=0^{\circ}$ and $\theta=90^{\circ}$, that corresponds to the $\mathrm{B}_{3,0}$ conformation. The more detailed study shows that, for crystalline cellulose, almost all the rings are ${ }^{4} \mathrm{C}_{1}$ conformers. However, in the amorphous (paracrystalline) phase, the percentage of ${ }^{4} \mathrm{C}_{1}$ conformations decreases to $63 \%(64 \%)$, and, additionally, new conformations show up: ${ }^{1} \mathrm{C}_{4}$ with $19 \%(25 \%)$ and boat or skew with $18 \%(11 \%)$. This analysis reveals that puckering in crystalline and noncrystalline cellulose shows important differences, whereas the paracrystalline and fully amorphous phases differ in the number of boat conformers. More boat conformers in amorphous cellulose can be related to less ordered structure and entangled chains. Some of these differences were also obtained by (Mazeau and Heux 2003). Interestingly, the percentage of boat conformations (11-18\%) is near the occurrence of the $60^{\circ}$ mode in $\Phi$ and $\Psi$ distributions (5-10\%), which suggests that these two phenomena can be are related and occur as e result of severe changes in chain direction. The authors realize, that the presented analysis may be force field dependent and a result that arises from the use of the GROMOS 53a6 force field which not optimized for puckering, and thus may not necessarily be confirmed via experiments (Nishiyama et al. 2002b; Dowd et al. 1994).

\section{Radial distribution function}

The radial distribution function (RDF), $g(r)$, is the probability of finding a pair of atoms of given type at a distance $r$. The RDF of crystalline, paracrystalline and amorphous cellulose provides a detailed insight into the extent of the long range ordering in these phases (Fig. 6). In particular, we quantify the probability of finding an atom within a given distance from a chosen atom, in this case pair correlation between $\mathrm{C} 4$ carbon atoms in the cellulose backbone. The RDF curve of amorphous cellulose is the average over RDF curves of the 5 different amorphous cellulose structures. The Fig. 6 shows that only the first maxima of RDF at about $5 \AA$ are overlapping for each cellulose phase. At longer distances, the peaks of amorphous cellulose are much broader, which demonstrates that the long-range ordering diminishes beyond $5 \AA$ distance. Moreover, the second maximum on the RDF of amorphous cellulose is positioned at a higher distance than that of 


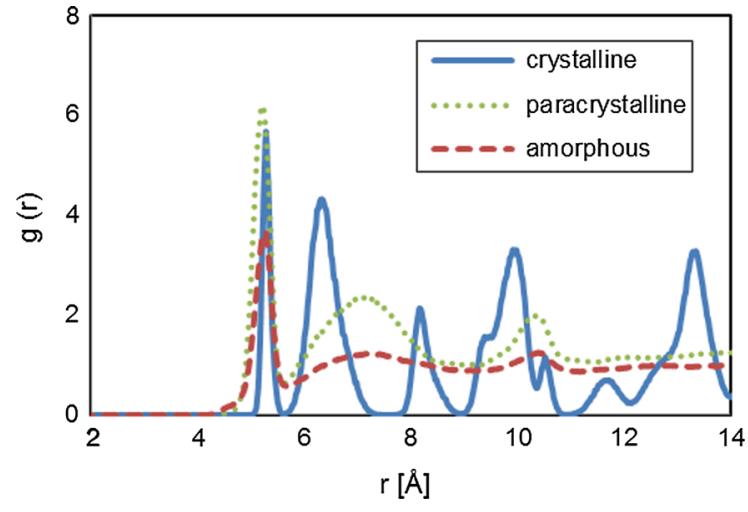

Fig. 6 The radial distribution function of C4-C4 pair correlation, plotted for crystalline, paracrystalline and amorphous cellulose

crystalline, which indicates larger spacing between the chains.

The RDF of the paracrystalline cellulose shows that the long range ordering is lost, however the peaks intensity is still in between that of corresponding amorphous and cellulose peaks. This result is corroborated by the torsion angles distribution presented in Fig. 4. Moreover, one can observe a shift in the position of the second maximum on the RDF when going from crystalline to paracrystalline and amorphous. The second peak, corresponding to the distance to the closest glucose monomer from different chain, the value of $r$ is respectively 6.32, 7.18 and 7.26. One should notice that some peaks of the crystalline RDF are 'merged' to a single broad peak in the case of paracrystalline and amorphous, thus in the presented range the RDF of non-crystalline phases have only three discernable peaks.

Another MD study (Mazeau and Heux 2003) where the RDF of a crystalline and amorphous cellulose was presented taking into account every atom pair, in contrast to our paper where we compare $\mathrm{C} 4-\mathrm{C} 4$ pair correlation, cannot be directly compared. However, we note that the characters of the RDF of amorphous cellulose are the same, relatively uniform, without significant peaks after the distance of larger than one glucopyranose unit.

\section{Potential energy}

The structural models of crystalline, paracrystalline and amorphous cellulose were quenched to and equilibrated at $300 \mathrm{~K}$ in order to examine the differences in the total potential energy and in structure of the different cellulose polymorphs. In Fig. 7a the total potential energy is broken down into: bonded (bonds, angles, dihedrals and impropers), nonbonded (short- and long-range Lennard-Jones interactions) and electric (short- and long-range Coulomb energy) interactions, for crystalline, paracrystalline and amorphous cellulose. The error bars for amorphous cellulose are the standard deviations that come from different amorphous cellulose structures run with the same parameters. The results show that the total energies of paracrystalline $\left(343 \mathrm{~kJ} \mathrm{~mol}^{-1}\right)$ and amorphous cellulose $\left(348 \mathrm{~kJ} \mathrm{~mol}^{-1}\right)$ are roughly equal and are slightly above that of crystalline cellulose $\left(342 \mathrm{~kJ} \mathrm{~mol}^{-1}\right)$.

The amorphous phase exhibits lower energy of bonded interaction, which is compensated by higher non-bonded and Coulomb interactions. The lower energy of bonded interaction is a result of the process of obtaining amorphous cellulose: high temperature and large spacing enable the chains to adjust their bonds and angles to the minimum energy conformation, resulting in chain recoil and twisting. On the other hand, larger inter-chain spacing in these systems give rise to an increase in both electrostatic and Lennard-Jones interaction energy. One should notice that, for each contributing type of energy, the energy values of paracrystalline cellulose always show a trend between the ones for amorphous and crystalline states, which suggests that paracrystalline is a non-equilibrium intermediate structure. We note that Mazeau and Heux (2003) obtained an amorphous cellulose model by inserting in the box one cellulose residue after another and randomly attributing $\omega$ and $\Psi$ torsion angles, and reported comparisons of the potential energy. In their study, all of the energy components of amorphous cellulose were found to be higher than those of crystalline cellulose, including bonded potentials. Contrary to this observation, we find that the energy of bonded interactions of crystalline domains is actually higher to the energy of interaction of amorphous domains, which is reasonable when considering that these chains are more extended and thus will have higher bond energy. The discrepancy with respect to the previous numerical studies of cellulose could be attributed to the differences in force fields used (PCFF, all-atom) or relaxation state of the amorphous domains. Our modelling approach of amorphous cellulose is based on high-temperature equilibration, 

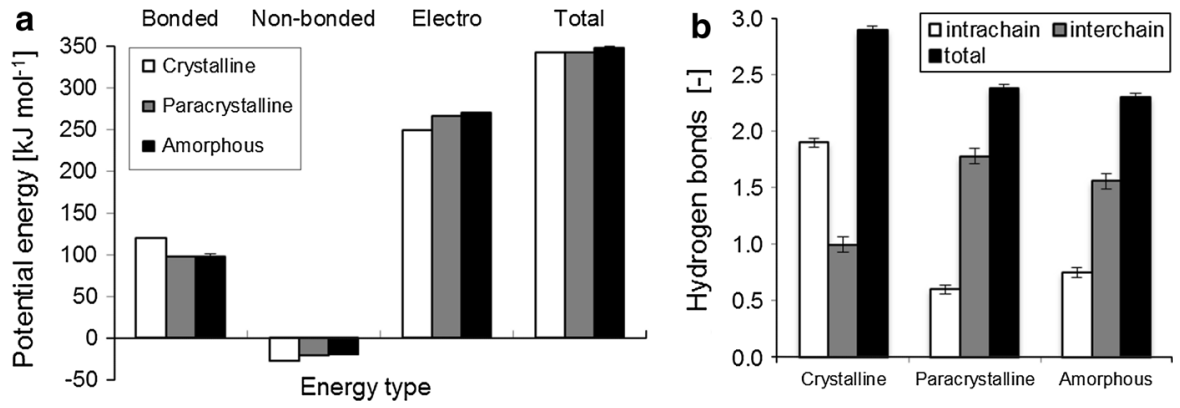

Fig. 7 a Comparison of total potential energy and contributing bonded, non-bonded and Coulomb interaction energies for three states of cellulose: crystalline, paracrystalline and amorphous. Values are given at $\mathrm{T}=300 \mathrm{~K}$ and are normalized by the

which facilitated overcoming kinetic barriers and helps to obtain lower energy conformations of glucose units. The error values for amorphous cellulose stress come from the statistics obtained from 5 different simulations that give rise to slightly different structures.

\section{Thermal expansion coefficients}

We examine next the mechanical properties. Table 2 contains the thermal expansion coefficients (TEC) for each cellulose state. The TEC are valid in the range 250-350 K, where the expansion is linear for crystalline cellulose. Above $400 \mathrm{~K}$ the expansion of crystalline cellulose starts to be non-linear, the threshold being close to $470 \mathrm{~K}$ as found by (Hori and Wada 2005). The values in ' $a$ ' direction and the volumetric TEC are close to the ones obtained experimentally (Hori and Wada 2005). One can notice a significant anisotropy of TECs in different directions of the crystalline cellulose lattice. The anisotropy is due to the different bonding types along each lattice direction, as discussed above, and is related to the data presented in Fig. 2c, that show different character of temperature profiles between ' $a$ ' or ' $b$ ' (decrease only in the transition region, increase otherwise) and 'c' (always decreases). As the inter-chain distance increases due to thermal motion, the stretched cellulose chains are able to reach lower energy state by shrinking, which in turn results in a negative TEC in 'c' direction.

The smaller volumetric expansion coefficient of the amorphous cellulose is a result of its porous structure: an increase in temperature allows the chains to expand toward their inter-chain space rather than outwards. number of glucose monomers. b Intramolecular, intermolecular and total number of hydrogen bonds per glucose units for crystalline, paracrystalline and amorphous cellulose

This is not the case for crystalline; its structure is nonporous and the space between the cellulose sheets is $<1 \mathrm{~nm}$, therefore its TEC is greater than that of amorphous cellulose.

\section{Elastic modulus}

Now we study Young's modulus values along each lattice directions of crystalline cellulose, paracrystalline and the average modulus of amorphous cellulose, which values are given in Table 3 . The elastic moduli of the crystalline cellulose obtained in our study are in good agreement with experiments (Tashiro and Kobayashi 1991), for example, the modulus along c-axis, obtained by X-ray diffraction, is typically in the range 100-160 GPa (Eichhorn et al. 2010). In their study, (Wohlert et al. 2012) used GROMOS force field to quantify the elastic modulus and obtained a value of $113 \mathrm{GPa}$, that is lower than ours. This difference can be attributed to finite chains and the presence of water. Crystalline cellulose is strongly anisotropic. The anisotropy of the elastic properties of crystalline cellulose $\mathrm{I} \beta$ from first principles density functional theory with dispersion correction was reported in (Dri et al. 2013). As mentioned above, this difference comes from the different bonding types. The largest modulus was measured in $c$ direction and is a result of strong covalent bonds. The weakest bonding by van der Waals forces is reflected by the lowest modulus value in $a$ direction. On the other hand, the elastic modulus of amorphous cellulose averaged over all directions has value $5.6 \mathrm{GPa}$, which is even smaller than the modulus of the crystalline cellulose in the $a$ direction $(11.3 \mathrm{GPa})$. The a-axis modulus is higher 
Table 2 Thermal expansion coefficients of crystalline cellulose, along the lattice directions, and of paracrystalline and amorphous state, averaged over box dimensions, due to isotropy

Volumetric expansion coefficient are given for comparison

Table 3 Young's modulus of crystalline cellulose, given for each lattice direction, paracrystalline cellulose, for crosssectional and longitudinal directions, and of amorphous phase, averaged over three directions

\begin{tabular}{llccc}
\hline Model & \multicolumn{3}{l}{ Thermal expansion coefficient } \\
\cline { 2 - 5 } & $\mathrm{a}\left(\times 10^{-5} \mathrm{~K}^{-1}\right)$ & $\mathrm{b}\left(\times 10^{-5} \mathrm{~K}^{-1}\right)$ & $\mathrm{c}\left(\times 10^{-5} \mathrm{~K}^{-1}\right)$ & $\begin{array}{c}\text { volumetric } \\
\left(\times 10^{-5} \mathrm{~K}^{-1}\right)\end{array}$ \\
\hline Crystalline & 10.8 & 0.91 & -0.68 & 11.3 \\
$\begin{array}{l}\text { Crystalline experimental } \\
\text { (Hori and Wada 2005) }\end{array}$ & 13.6 & -3.0 & 0.6 & 11.1 \\
Paracrystalline & 6.04 & & 1.57 & 10.87 \\
Amorphous & $2.8 \pm 1.7$ & & $6.6 \pm 0.5$ \\
\hline
\end{tabular}

\begin{tabular}{llll}
\hline Model & \multicolumn{2}{l}{ Young's modulus $(\mathrm{GPa})$} & \\
\cline { 2 - 4 } & $\mathrm{a}$ & $\mathrm{b}$ & $\mathrm{c}$ \\
\hline $\begin{array}{l}\text { Crystalline [experimental } \\
\text { (Tashiro and Kobayashi }\end{array}$ & $11.3(15.16)$ & $72.6(54.55)$ & $150.4(167.79)$ \\
$\begin{array}{l}\text { 1991)] } \\
\text { Paracrystalline }\end{array}$ & & \\
Amorphous & 3.0 & & 33.5 \\
\hline
\end{tabular}

in the crystalline phase because of higher packing of cellulose sheets, which increases the density and effective modulus, the collective direction of the van der Waals stacking interactions and the amphiphilic sheet interactions that arise from the ordering of the sheets, which is lost upon loss of crystalline structure (Sinko et al. 2013). This finding suggests that van der Waals bonds are the ones that are mostly distorted and the main strain contributors, when amorphous cellulose is under tension or compression. The obtained Young's modulus value of amorphous phase is lower than $10.42 \mathrm{GPa}$ that was obtained by MD in (Chen et al. 2004a, b), and $8.45 \mathrm{GPa}$, that was reported for viscose rayon experiments (Chen et al. 2004b). The average value of Young's modulus for paracrystalline phase in cross-sectional directions $(3.0 \mathrm{GPa})$ is smaller than average modulus of amorphous cellulose. This is the result of the fact that in the cross-sectional plane only non-bonded interactions contribute to the strain. This finding confirms that chain ordering has a strong influence on the material stiffness.

The smaller modulus of amorphous cellulose correlates with the lower value of total number of hydrogen bonds per glucose unit (Fig. 7b). This value is divided into intramolecular (within a chain) and intermolecular (between the chains) hydrogen bonds. The obtained values suggest that the largest change occurs in the intermolecular bonding system, which leads to larger spacing between chains and thus creates porous structure of amorphous cellulose. In crystalline cellulose, the number of hydrogen bonds within chains is about twice than the one between chain, in agreement with experiment (Nishiyama et al. 2002b). However, during amorphisation, the number of intrachain hydrogen bonds substantially decreases in favor of interchain hydrogen bonds. This comes from chain twisting and breaking up the wellorganized sheet structure. In the case of paracrystalline, the average number of hydrogen bonds, 2.43 , is between the values 2.97 and 2.31 for crystalline and amorphous, respectively. This correlates with the value of elastic modulus ( $35.3 \mathrm{GPa})$, being between the corresponding values of crystalline and amorphous cellulose: 150.4 and 5.6 GPa, respectively (Table 3). The number of hydrogen bonds in paracrystalline is closer to that of amorphous cellulose, which suggests that the large number of hydrogen bonds in crystalline cellulose is due to near perfect chain alignment in sheets that decreases quickly at elevated temperatures.

\section{Conclusion}

The three cellulose allomorphs: crystalline I $\beta$, paracrystalline and amorphous, have been constructed and studied in this paper using all-atoms MD simulations.

Equilibration of the crystalline cellulose at an elevated temperature, followed by quenching, resulted 
in the formation of paracrystalline cellulose. The pathway to a paracrystalline state is certainly not unique, but the obtained physical features are distinct (e.g. anisotropy and elastic modulus) from both crystalline and amorphous phases. The obtained model is an example paracrystalline structure that captures partial ordering effect and might be relevant to the surface chains of cellulose microfibrils. By comparing the mechanical properties, dihedral distribution, hydrogen bonding, chain ordering and potential energy, the paracrystalline cellulose was shown to be an intermediary state between crystalline and amorphous cellulose. Grasping the key differences between different states of cellulose will help to understand the amorphisation process and structure at interfaces between crystalline and amorphous phases of cellulose in complex biological structures. The amorphous phase was obtained by high temperature equilibration of randomly arranged cellulose chains. The high temperature compensates the short simulation time, characteristic for MD simulations, and help to find minimum energy state without being trapped in local minima. In this way the amorphous cellulose structure is a complex dynamic phase in slow evolution, similar to glassy solids.

The structure of amorphous cellulose obtained in this work corroborates experimental findings on the mechanical and topological characteristics. The main criterion to distinguish between the three cellulose phases is their mechanical properties: whereas the crystalline cellulose has distinct elastic moduli in three different directions, only two distinct elastic moduli in the paracrystalline phase can be measured corresponding to the directions along and normal to the cellulose chains, and amorphous cellulose is indeed mechanically isotropic.

We anticipate that the hydration will significantly lower mechanical properties such as the elastic modulus, and induce swelling in amorphous cellulose. However, crystalline cellulose should remain dry due to its impenetrable core structure, but may be affected at its surfaces. Paracrystalline is expected to behave as an intermediate scenario between the two extremes. Future studies will consider these effects on the structure and mechanics of cellulose systems at the nanoscale. The importance of non-crystalline cellulose comes from being an interface between crystalline cellulose and semicrystalline hemicellulose phases in a microfibril model. The main contribution of this paper is the establishment of the structural and mechanical characteristics of the three distinct phases of cellulose, which paves the way for multi-scale studies on the mechanics of wood and cellulose based materials.

Acknowledgments The authors are grateful for the financial support of the Swiss National Science Foundation (SNF) under Grants No. 125184 and 143601. S.K. acknowledges support from the Army Research Office (Grant \# W911 NF-13-1-0241).

\section{References}

Agarwal UP (2006) Raman imaging to investigate ultrastructure and composition of plant cell walls: distribution of lignin and cellulose in black spruce wood (Picea mariana). Planta 224(5):1141-1153. doi:10.1007/s00425-006-0295-Z

Andersson S, Serimaa R, Paakkari T, Saranpaa P, Pesonen E (2003) Crystallinity of wood and the size of cellulose crystallites in Norway spruce (Picea abies). J Wood Sci 49(6):531-537. doi:10.1007/s10086-003-0518-x

Apol E, Apostolov R, Berendsen H, Van Buuren A, Bjelkmar P, Van Drunen R, Feenstra A, Groenhof G, Kasson P, Larsson P (2010) GROMACS user manual version 4.5. 4. Royal Institute of Technology and Uppsala University, Stockholm

Berendsen HJ, Postma JPM, van Gunsteren WF, DiNola A, Haak J (1984) Molecular dynamics with coupling to an external bath. J Chem Phys 81(8):3684-3690

Bergenstrahle M, Berglund LA, Mazeau K (2007) Thermal response in crystalline I beta cellulose: a molecular dynamics study. J Phys Chem B 111(30):9138-9145. doi:10.1021/Jp072558i

Bergenstrahle M, Mazeau K, Berglund LA (2008a) Molecular modeling of interfaces between cellulose crystals and surrounding molecules: effects of caprolactone surface grafting. Eur Polym J 44(11):3662-3669. doi:10.1016/j. eurpolymj.2008.08.029

Bergenstrahle M, Wohlert J, Larsson PT, Mazeau K, Berglund LA (2008b) Dynamics of cellulose-water interfaces: NMR spin-lattice relaxation times calculated from atomistic computer simulations. J Phys Chem B 112(9):2590-2595. doi: $10.1021 / \mathrm{Jp} 074641 \mathrm{t}$

Bergenstrahle M, Wohlert J, Himmel ME, Brady JW (2010) Simulation studies of the insolubility of cellulose. Carbohydr Res 345(14):2060-2066. doi:10.1016/j.carres.2010. 06.017

Bussi G, Donadio D, Parrinello M (2007) Canonical sampling through velocity rescaling. J Chem Phys 126(1):014101

Charlier L, Mazeau K (2012) Molecular modeling of the structural and dynamical properties of secondary plant cell walls: influence of lignin chemistry. J Phys Chem B 116(14):4163-4174. doi:10.1021/Jp300395k

Chen W, Lickfield GC, Yang CQ (2004a) Molecular modeling of cellulose in amorphous state part II: effects of rigid and flexible crosslinks on cellulose. Polymer 45(21):73577365. doi:10.1016/j.polymer.2004.08.023 
Chen W, Lickfield GC, Yang CQ (2004b) Molecular modeling of cellulose in amorphous state. Part I: model building and plastic deformation study. Polymer 45(3):1063-1071. doi:10.1016/j.polymer.2003.11.020

Chen W, Wang T, Lickfield GC (2007) Molecular modeling of cellulose in amorphous state. III. An innovative elastomeric crosslink system. J Polym Sci Polym Phys 45(14):1821-1833. doi:10.1002/Polb.21214

Chen P, Nishiyama Y, Mazeau K (2012) Torsional entropy at the origin of the reversible temperature-induced phase transition of cellulose. Macromolecules 45(1):362-368. doi:10.1021/Ma201954s

Chen P, Nishiyama Y, Putaux J-L, Mazeau K (2013) Diversity of potential hydrogen bonds in cellulose I revealed by molecular dynamics simulation. Cellulose. doi:10.1007/ s10570-013-0053-x

Cheng Q, Wang S, Harper DP (2009) Effects of process and source on elastic modulus of single cellulose fibrils evaluated by atomic force microscopy. Compos Part A: Appl Sci Manuf 40(5):583-588

Cremer D, Pople JA (1975) General definition of ring puckering coordinates. J Am Chem Soc 97(6):1354-1358

Deguchi S, Tsujii K, Horikoshi K (2006) Cooking cellulose in hot and compressed water. Chem Commun 31:3293-3295. doi: $10.1039 / \mathrm{b} 605812 \mathrm{~d}$

Deguchi S, Tsujii K, Horikoshi K (2008) Crystalline-to-amorphous transformation of cellulose in hot and compressed water and its implications for hydrothermal conversion. Green Chem 10(2):191-196. doi:10.1039/B713655B

Derome D, Rafsanjani A, Patera A, Guyer R, Carmeliet J (2012) Hygromorphic behaviour of cellular material: hysteretic swelling and shrinkage of wood probed by phase contrast X-ray tomography. Philos Mag 92(28-30):3680-3698. doi:10.1080/14786435.2012.715248

Dinwoodie JM, Building Research Establishment (2000) Timber, its nature and behaviour, 2nd edn, E \& FN Spon, BRE, with the support of the Centre for Timber Technology and Construction at BRE, London, New York, England

Dowd MK, French AD, Reilly PJ (1994) Modeling of aldopyranosyl ring puckering with MM3 (92). Carbohydr Res 264(1):1-19

Dri FL, Hector LG, Moon RJ, Zavattieri PD (2013) Anisotropy of the elastic properties of crystalline cellulose I-beta from first principles density functional theory with Van der Waals interactions. Cellulose 20(6):2703-2718. doi:10. 1007/s10570-013-0071-8

Eichhorn SJ (2012) Stiff as a board: perspectives on the crystalline modulus of cellulose. ACS Macro Lett 1(11): 1237-1239

Eichhorn S, Young R (2001) The Young's modulus of a microcrystalline cellulose. Cellulose 8(3):197-207

Eichhorn S, Dufresne A, Aranguren M, Marcovich N, Capadona J, Rowan S, Weder C, Thielemans W, Roman M, Renneckar S (2010) Review: current international research into cellulose nanofibres and nanocomposites. J Mater Sci 45(1):1-33

Fink H-P, Philipp B, Paul D, Serimaa R, Paakkari T (1987) The structure of amorphous cellulose as revealed by wide-angle X-ray scattering. Polymer 28(8):1265-1270

Gomes TCF, Skaf MS (2012) Cellulose-builder: a toolkit for building crystalline structures of cellulose. J Comput Chem 33(14):1338-1346. doi:10.1002/Jcc.22959
Hadden JA, French AD, Woods RJ Effect of microfibril twisting on theoretical powder diffraction patterns of cellulose I $\beta$. Cellulose. doi:10.1007/s10570-013-0051-z

Hardy BJ, Sarko A (1996) Molecular dynamics simulations and diffraction-based analysis of the native cellulose fibre: structural modelling of the I- $\alpha$ and I- $\beta$ phases and their interconversion. Polymer 37(10): 1833-1839

Hess B, Kutzner C, van der Spoel D, Lindahl E (2008) GROMACS 4: algorithms for highly efficient, load-balanced, and scalable molecular simulation. J Chem Theory Comput 4(3):435-447

Heymann B, Grubmüller H (1999) Elastic properties of poly (ethylene-glycol) studied by molecular dynamics stretching simulations. Chem Phys Lett 307(5):425-432

Hori R, Wada M (2005) The thermal expansion of wood cellulose crystals. Cellulose 12(5):479-484

Langan P, Nishiyama Y, Chanzy H (1999) A revised structure and hydrogen-bonding system in cellulose II from a neutron fiber diffraction analysis. J Am Chem Soc 121(43):9940-9946

Liao RJ, Zhu MZ, Zhou X, Zhang FZ, Yan JM, Zhu WB, Gu C (2012) Molecular dynamics study of the disruption of $\mathrm{H}$-bonds by water molecules and its diffusion behavior in amorphous cellulose. Mod Phys Lett B 26 (14), Artn 1250088. doi:10.1142/S0217984912500881

Lins RD, Hünenberger PH (2005) A new GROMOS force field for hexopyranose-based carbohydrates. J Comput Chem 26(13):1400-1412

Matthews JF, Skopec CE, Mason PE, Zuccato P, Torget RW, Sugiyama J, Himmel ME, Brady JW (2006) Computer simulation studies of microcrystalline cellulose I beta. Carbohydr Res 341(1):138-152. doi:10.1016/j.carres. 2005.09.028

Matthews JF, Beckham GT, Bergenstrahle-Wohlert M, Brady JW, Himmel ME, Crowley MF (2012) Comparison of cellulose I beta simulations with three carbohydrate force fields. J Chem Theory Comput 8(2):735-748. doi:10.1021/ Ct2007692

Mazeau K, Heux L (2003) Molecular dynamics simulations of bulk native crystalline and amorphous structures of cellulose. J Phys Chem B 107(10):2394-2403. doi:10.1021/ Jp0219395

Moon RJ, Martini A, Nairn J, Simonsen J, Youngblood J (2011) Cellulose nanomaterials review: structure, properties and nanocomposites. Chem Soc Rev 40(7):3941-3994

Muller M, Czihak C, Burghammer M, Riekel C (2000) Combined $\mathrm{X}$-ray microbeam small-angle scattering and fibre diffraction experiments on single native cellulose fibres. J Appl Crystallogr 33(3):817-819

Nishiyama Y (2009) Structure and properties of the cellulose microfibril. J Wood Sci 55(4):241-249. doi:10.1007/ s10086-009-1029-1

Nishiyama Y, Chanzy H, Langan P (2002a) Neutron and synchrotron $\mathrm{x}$-ray fiber diffraction studies of cellulose polymorphs. Abstr Pap Am Chem Soc 223:D26-D26

Nishiyama Y, Langan P, Chanzy H (2002b) Crystal structure and hydrogen-bonding system in cellulose 1 beta from synchrotron X-ray and neutron fiber diffraction. J Am Chem Soc 124(31):9074-9082. doi:10.1021/Ja0257319

Nishiyama Y, Sugiyama J, Chanzy H, Langan P (2003) Crystal structure and hydrogen bonding system in cellulose $\mathrm{I} \alpha$ 
from synchrotron X-ray and neutron fiber diffraction. J Am Chem Soc 125(47):14300-14306

Oostenbrink C, Villa A, Mark AE, Van Gunsteren WF (2004) A biomolecular force field based on the free enthalpy of hydration and solvation: the GROMOS force-field parameter sets 53A5 and 53A6. J Comput Chem 25(13):1656-1676

Rief M, Oesterhelt F, Heymann B, Gaub HE (1997) Single molecule force spectroscopy on polysaccharides by atomic force microscopy. Science 275(5304):1295-1297

Sinko R, Mishra S, Ruiz L, Brandis N, Keten S (2013) Dimensions of biological cellulose nanocrystals maximize fracture strength. ACS Macro Lett 3:64-69

Sugiyama J, Persson J, Chanzy H (1991a) Combined infrared and electron diffraction study of the polymorphism of native celluloses. Macromolecules 24(9):2461-2466

Sugiyama J, Vuong R, Chanzy H (1991b) Electron diffraction study on the two crystalline phases occurring in native cellulose from an algal cell wall. Macromolecules 24(14):4168-4175

Tanpichai S, Quero F, Nogi M, Yano H, Young RJ, Lindström T, Sampson WW, Eichhorn SJ (2012) Effective Young's modulus of bacterial and microfibrillated cellulose fibrils in fibrous networks. Biomacromolecules 13(5):1340-1349

Tashiro K, Kobayashi M (1991) Theoretical evaluation of threedimensional elastic constants of native and regenerated celluloses: role of hydrogen bonds. Polymer 32(8):1516-1526

Wada M, Okano T, Sugiyama J (2001) Allomorphs of native crystalline cellulose I evaluated by two equatoriald-spacings. J Wood Sci 47(2):124-128

Wada M, Chanzy H, Nishiyama Y, Langan P (2004) Cellulose IIII crystal structure and hydrogen bonding by synchrotron $\mathrm{X}$-ray and neutron fiber diffraction. Macromolecules 37(23):8548-8555

Wohlert J, Bergenstrahle-Wohlert M, Berglund LA (2012) Deformation of cellulose nanocrystals: entropy, internal energy and temperature dependence. Cellulose 19(6):18211836. doi:10.1007/s10570-012-9774-5

Zhang Q, Bulone V, Ågren H, Tu Y (2011) A molecular dynamics study of the thermal response of crystalline cellulose I $\beta$. Cellulose 18(2):207-221 